\title{
Regge description of high energy pion pion total cross sections
}

\author{
José R. Peláez \\ Departamento de Física Teórica II. Universidad Complutense. 28040 Madrid. Spain.
}

September 23, 2013

\begin{abstract}
We have recently presented a Regge description of $\pi \pi$ total cross sections valid above $1.4 \mathrm{GeV}$, consistent with the few existing experiments, factorization and crossing symmetry. In this note we show how it also describes a further large data sample obtained from an analysis of experiments on $\pi^{ \pm} p \rightarrow X \Delta^{++}$and $\pi^{ \pm} n \rightarrow X p$.
\end{abstract}

\section{Regge description of $\pi \pi$ total cross sections}

In references 1, 2], we have shown how it was possible to obtain a precise Regge description of high energy total $\pi \pi$ scattering down to $E_{k i n} \simeq 1.1 \mathrm{GeV}$. Apart from the interest in itself, there has been a renewed interest in this high energy region because the imaginary part of the $\pi \pi \rightarrow \pi \pi$ amplitude is needed for dispersive studies aiming at a precise description of $\pi \pi$ data at low energies [3, 4, 1].

A relevant property of our description is that it respects factorization. This means that, for instance, the imaginary part of an amplitude $F_{A+B \rightarrow A+B}$ is:

$$
\operatorname{Im} F_{A+B \rightarrow A+B}(s, t) \simeq f_{A}(t) f_{B}(t)(s / \hat{s})^{\alpha_{R}(t)}, \quad \hat{s}=(1 \mathrm{GeV})^{2} .
$$

The $(s / \hat{s})^{\alpha_{R}(t)}$ behavior comes from the so-called Regge pole $R$. All poles have $\alpha_{R}<1$ and thus vanish for large $s$, except the Pomeron that scales like $s$ up to around 15 or $20 \mathrm{MeV}$, where it dominates all other pole contributions, giving a common prediction $\sigma^{\infty}$ for all $\pi \pi$ channels. For larger energies it increases logarithmically. As a matter of fact there could be many Regge poles exchanged in each channel, all them with their corresponding $f_{i}(t)$ factors depending on $R$ and the particles in the initial state. Using factorization, it is thus possible to obtain the $\pi \pi$ Regge amplitudes from those of $\pi N$ and $N N$. Total cross sections are then related to forward scattering amplitudes by: $\sigma_{A B}=4 \pi^{2} \operatorname{Im} F_{A+B \rightarrow A+B}(s, 0) / \lambda^{1 / 2}\left(s, m_{A}^{2}, m_{B}^{2}\right)$, with $\lambda(a, b, c)=a^{2}+b^{2}+c^{2}-2 a b-2 a c-2 b c$. Thus we 2] fitted the large $\pi^{ \pm} N$ and $N N$ data compilation of the COMPASS group as given in the Particle Data Tables [5], and the few $\pi \pi$ data [6] points known to us down to $E_{k i n} \simeq 1.1$ imposing factorization. The fit parameters are largely dominated by the $\pi^{ \pm} N$ and $N N$ experiments, but still we obtained a very precise description for $\pi \pi$ total cross sections, that was in remarkable agreement with the $\sigma_{\text {tot }}^{\pi \pi}$ data above $2 \mathrm{GeV}$. At lower energies these data are in conflict with the $\sigma_{t o t}^{\pi \pi}$ reconstructed 2] from lower energy phase shifts analysis and our results fall somewhere in-between. We refer to our paper [2] for further details.

In addition, we have also checked that our high energy results together with fits [1] to the low energy satisfy two crossing symmetry sum rules. This is again of relevance because in the seventies [7] there was a suggestion that the predictions of factorization $\sigma^{\infty} \simeq 13 \mathrm{mb}$, together with the existing phase shifts analysis at that time, violated crossing symmetry, suggesting $\sigma^{\infty}=6 \pm 5 \mathrm{mb}$. Of course this was tenable until the first high energy $\sigma_{\text {tot }}^{\pi \pi}$ were measured, and indeed the very same authors [7] pointed out somewhat later that the central value should be raised to $\sigma^{\infty}=8.3 \mathrm{mb}$. The recent studies in [3, 4] used $\sigma^{\infty}=5 \pm 3 \mathrm{mb}$, following [3]. Unfortunately, the $\sigma_{\text {tot }}^{\pi \pi}$ data went largely unnoticed to our days, including to ourselves, so that in [1] the use of factorization was only based on QCD considerations. In 2] we "rediscovered" four different experimental works [6] that we used in a reanalysis to find $\sigma_{\text {tot }}(20 \mathrm{GeV})=$ $13.4 \pm 0.6 \mathrm{mb}$, while simultaneously respecting crossing.

To appear in the proceedings of MESON2004, Krakow, Poland, July 2004. 


\section{Comparison with further data}

Following the discussions of my talk on this MESON2004 conference I came to know that there was another analysis [8] of $\pi \pi$ total cross sections. In that work, a triple reggeon model is used to analyze several sets of experimental data on $p p \rightarrow X \Delta^{++}$and $p n(p) \rightarrow X p(n)$, and obtain Regge parameters with whom to extract total $\pi^{ \pm} \pi^{-}$cross sections from $\pi^{ \pm} p \rightarrow X \Delta^{++}$and $\pi^{ \pm} n \rightarrow X p$. The most relevant contribution of this paper is the inclusion of absorptive corrections in the last two reactions, which seems to decrease the results by about 10 to 15\%. In Fig.1, we show how our Regge description, and in particular, our value $\sigma_{\text {tot }}(20 \mathrm{GeV})=13.4 \pm 0.6 \mathrm{mb}$ indeed provides a good description of this data, which strongly disfavors a value more than two times smaller. Following the authors we display only the statistical errors. Systematic errors were estimated at the $7-10 \%$ level.

\section{Acknowledgments}

I thank the MESON2004 organizers for the stimulating workshop, since this note originated in the discussion following my talk. In particular, I thank A. Szczurek and N.N. Nikolaev for their comments on the data 8]. I also thank F.J. Ynduráin for comments and suggestions and as coauthor of the theoretical Regge description reviewed here. I am very grateful for the hospitality of the Institut für Kernphysik (Theorie), Forschungzentrum Jülich, where this note was prepared. Financial support from Spanish CICYT projects BFM2000-1326, BFM2002-01003, and from the E.U. EURIDICE network HPRN-CT2002-00311 is also acknowledged.

\section{References}

[1] J. R. Peláez and F. J. Ynduráin, Phys. Rev. D68, 074005 (2003)

[2] J. R. Peláez and F. J. Ynduráin, Phys. Rev. D69, 114001 (2004)

[3] B.Ananthanarayan, G.Colangelo, J.Gasser and H.Leutwyler, Phys. Rept. 353, 207 (2001)

[4] G. Colangelo, J. Gasser and H. Leutwyler, Nucl. Phys. B 603, 125 (2001); S. Descotes-Genon, N. H. Fuchs, L. Girlanda and J. Stern, Eur. Phys. J. C 24, 469 (2002); R. Kaminski, L. Lesniak and B. Loiseau, Phys. Lett. B 551, 241 (2003); P. Buettiker, S. Descotes-Genon and B. Moussallam, Eur. Phys. J. C 33, 409 (2004).

[5] K. Hagiwara et al., Phys. Rev. D66 010001 (2002).

[6] Biswas, N. N., et al., Phys. Rev. Letters, 18, 273 (1967) $\left[\pi^{-} \pi^{-}, \pi^{+} \pi^{-}\right.$and $\left.\pi^{0} \pi^{-}\right]$; Cohen, D. et al., Phys. Rev. D7, 661 (1973) $\left[\pi^{-} \pi^{-}\right]$; Robertson, W. J., Walker, W. D., and Davis, J. L., Phys. Rev. D7, 2554 (1973) $\left[\pi^{+} \pi^{-}\right]$; Hoogland, W., et al. Nucl. Phys., B126, 109 (1977) $\left[\pi^{-} \pi^{-}\right]$; Hanlon, J., et al, Phys. Rev. Letters, 37, 967 (1976) $\left[\pi^{+} \pi^{-}\right]$; Abramowicz, H., et al. Nucl. Phys., B166, 62 (1980) $\left[\pi^{+} \pi^{-}\right]$.

[7] M. R. Pennington, Annals Phys. 92, 164 (1975). A. D. Martin and M. R. Pennington, Annals Phys. 114, 1 (1978).

[8] B.G. Zakharov and V.N. Sergeev, Sov. J. Nucl. Phys. 39, 448 (1984) also in Yad. Fiz. 39, 707 (1984). 

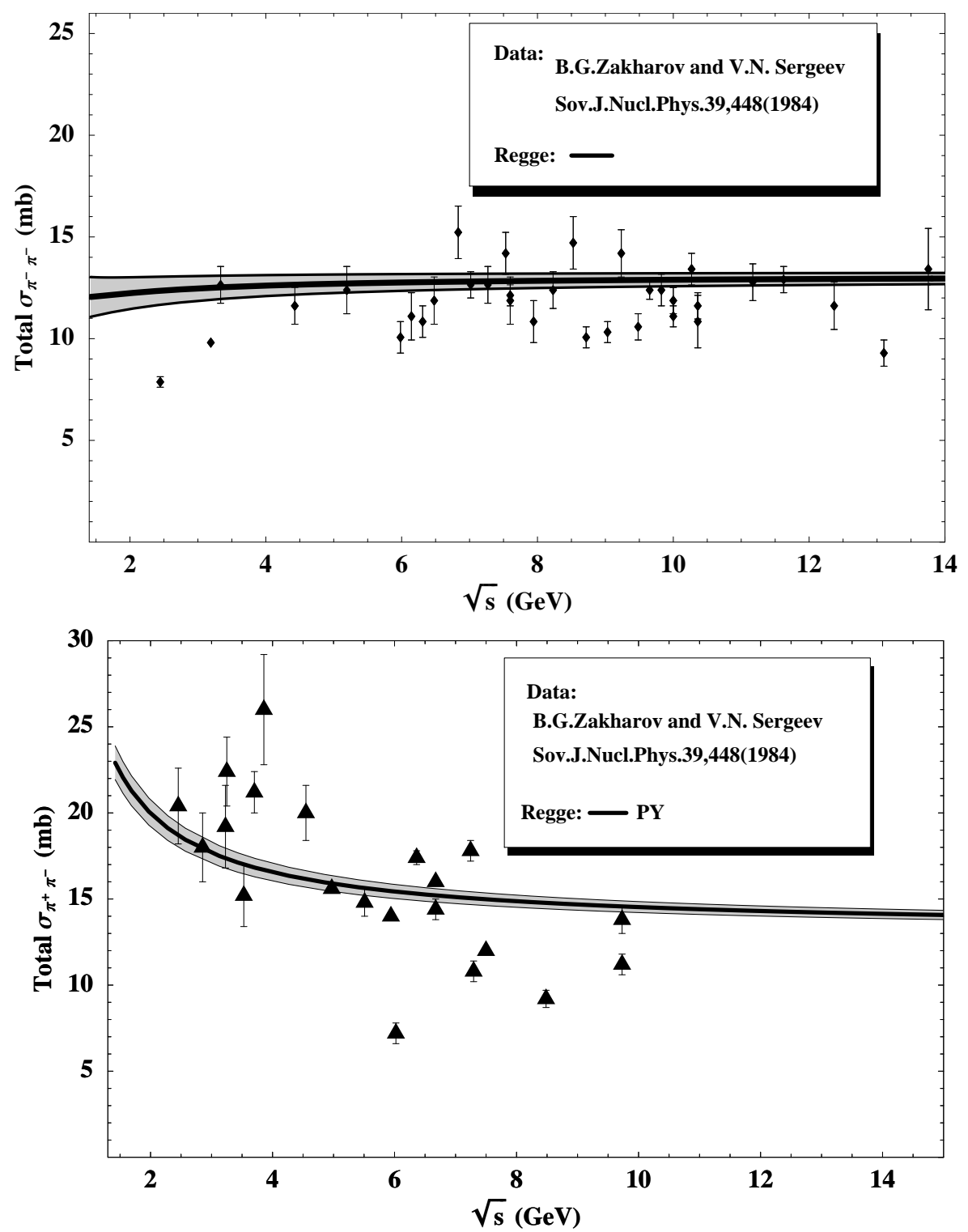

Figure 1: The continuous line stands for our Regge representation and the gray band for the associated uncertainty. Data are from [8] and the error bars are just statistical, however, the authors pointed out a "possible systematic error of $\simeq 7-10 \%$ ". 